\title{
Optimization and statistical analysis of $\mathrm{Au}-\mathrm{ZnO} / \mathrm{Al}_{2} \mathrm{O}_{3}$ catalyst for CO oxidation
}

\author{
Wenjin Yan ${ }^{\mathrm{a}, \mathrm{c}}$, Xinli Jia ${ }^{\mathrm{a}}$, Tao Chen ${ }^{\mathrm{b}, *}$, Yanhui Yang ${ }^{\mathrm{a}, *}$ \\ a. School of Chemical and Biomedical Engineering, Nanyang Technological University, \\ Singapore 637459; \\ b. Department of Chemical and Process Engineering, University of Surrey, Guildford GU2 \\ $7 X H, U K$; \\ c. Institute of Chemical \& Engineering Sciences, 1 Pesek Road, Jurong Island, Singapore
}

627833

\begin{abstract}
In our former work [Catal. Today 174 (2011) 127], 12 heterogeneous catalysts were screened for $\mathrm{CO}$ oxidation, and $\mathrm{Au}-\mathrm{ZnO} / \mathrm{Al}_{2} \mathrm{O}_{3}$ was chosen and optimized in terms of weight loading of $\mathrm{Au}$ and $\mathrm{ZnO}$. The present study follows on to consider the impact of process parameters (catalyst preparation and reaction conditions), in conjunction with catalyst composition (weight loading of $\mathrm{Au}$ and $\mathrm{ZnO}$, and total weight of the catalyst), as the optimization of the process parameters simultaneously optimized the catalyst composition. The optimization target is the reactivity of this important reaction. These factors were first optimized using response surface methodology (RSM) with 25 experiments, to obtain the optimum: $100 \mathrm{mg}$ of $1.0 \% \mathrm{Au}-4.1 \% \mathrm{ZnO} / \mathrm{Al}_{2} \mathrm{O}_{3}$ catalyst with 220 ${ }^{\circ} \mathrm{C}$ calcination and $100{ }^{\circ} \mathrm{C}$ reduction. After optimization, main effects and interactions of these five factors were studied using statistical sensitivity analysis (SA). Certain observations from SA were verified by reaction mechanism, reactivity test and/or characterization techniques, while others need further investigation.
\end{abstract}

Key words : carbon monoxide oxidation; data-based modeling; design of experiments; gold catalysts; heterogeneous catalysis; rapid optimization 
*Corresponding author Tao Chen: Tel: 0148368 6593; E-mail: t.chen@ @urrey.ac.uk

*Corresponding author Yanhui Yang : Tel: (+65)6316 8940; E-mail: yhyang@ntu.edu.sg

\section{Foundation item: This work was partially supported by the Singapore AcRF Tier 1 Grant (RG 19/09) and the A*STAR SERC Grant (102 1010020 ).}

\section{Introduction}

In catalysis study, carbon monoxide (CO) oxidation is widely used as a representative reaction to explore catalytic oxidation capability [1]. In addition, since $\mathrm{CO}$ is a highly noxious gas, high performance $\mathrm{CO}$ oxidation catalyst is needed in many applications, including gas mask, automobile exhaust treatment and indoor air quality control. There are also other applications requiring removal of $\mathrm{CO}$, such as polymer electrolyte membrane fuel cell [2] and $\mathrm{CO}_{2}$ laser [3]. The commonly used catalysts for this reaction include two categories: noble metal catalysts and those using transition metal oxides [4].

In our former work [5], the recipe was screened with noble metal-promoter/support combination, four choices for each component. The screening procedure chose $\mathrm{Au}-\mathrm{ZnO} / \mathrm{Al}_{2} \mathrm{O}_{3}$, after which modeling and optimization methods were applied to optimize the catalytic performance. However, in later study, we found that the conditions in catalyst preparation (e.g. calcination temperature) and reaction (e.g. catalyst amount) were also very important, since the optimization of them can further improve the composition of catalyst. In addition, optimized parameters can lead to better reaction activity and mechanism understanding. For this reason, a new project was investigated to optimize the process parameters simultaneously with catalyst composition. Five factors were selected: Au loading, $\mathrm{ZnO}$ loading, calcination temperature, $\mathrm{H}_{2}$ reduction temperature and catalyst weight.

The optimization structure is based on response surface methodology (RSM), which is a combination of design of experiments (DoE), modeling and optimization [6]. For DoE, 
Hammersley sequence sampling (HSS) method [7] was adopted due to its high uniformity and easy implementation [8]. In this study, HSS was applied to give experimental points a uniform distribution over the factors' space. For modeling, data-based modeling category was selected instead of first-principle and hybrid model, since the requirement of mechanism makes this framework restricted. Among data-based modeling methods, Gaussian process regression (GPR) approach [9] was chosen due to its superior and stable prediction performance [10]. With all the available data, GPR model was iteratively developed for this system. For optimization, since the existing highest reactivity was superior, the former expected improvement (EI) approach was not suitable. Alternatively, we adopted the classic optimization method which conducts experiment on the point with highest predictive mean. The optimization step repeated until the criterion was satisfied.

For a process, following the response optimization, the analysis of main effects and interactions are equally important. In this study, a statistical method, sensitivity analysis (SA), was implemented to analyze the process. A global SA approach, Sobol' SA method [11], was chosen due to its precise evaluation and low computation cost [12]. The analysis would study on the main effects and interactions of all the five factors, and then confirm them with thermodynamic rules or characterization results, if applicable. It should pay attention that the term "interaction" used in this paper is different from the physical one. Without specific mention, interaction in this paper would refer to the statistical sense. Nevertheless, statistical interaction is useful for physical interaction indication, where the latter one can only be evaluated with expensive and time-consuming characterization techniques.

The rest of this paper is organized as follows: Section 2 presents the overall introduction and implementation of RSM and SA methods. The details of catalyst synthesis and CO oxidation reaction are introduced in Section 3. The result of model-aided optimization and analysis are shown in Section 4, and then Section 5 concludes the whole study.

\section{Methods}


The overall framework of the model-aided optimization and analysis approach can be separated into four components:

1. DoE to allocate initial experimental points.

2. Development of data-based model from all available data.

3. Optimization using current data-based model, and allocate additional experimental point for model update.

4. Sensitivity analysis depends on final model.

In this framework, Step 1-3 combine as complete RSM. Step 2 and 3 form an iterative part, which stops after the optimization criterion is satisfied. Finally, Sobol' SA method analyzes the process based on the "newest" model. The introduction, choice and detail of the four parts are listed subsequently.

\subsection{Design of Experiments}

DoE methods are widely used in research and industry, to improve the speed and uniformity of space investigation, further saving time and cost [13-15]. The basic requirements of DoE are replication and randomization, so a good DoE technique should give reliable and uniform allocation. In experimental science, the using of DoE methods generally focus on fractional factorial design, central composite designs (CCD) and their variants. However, they had been proved to be weak for complex case [16]. A new concept, "space filling", was proposed to give a reliable cover for most of cases. The basic concept of "space filling" is to allocate all the sample points uniformly into the design spaces, so a straightforward solution is Monte Carlo random sampling. However, this approach requires a great number of sample points to reach a satisfactory uniformity, which is very costly and time-consuming. To overcome this disadvantage, stratified and deterministic sampling methods were investigated, such as Hammersley sequence sampling (HSS) [7], Latin hypercube sampling (LHS) [17] and uniform design (UD) [16]. In some comparison works, HSS and UD were demonstrated to have better uniformity than LHS [7]. In this study, HSS was selected since it attains easier implementation than UD. The details of HSS can be 
found in [7].

\subsection{Process Modeling}

Modeling part performs as a nexus in the whole RSM approach, as the uniformity of DoE will influence the accuracy of model, while the predictive ability of model determines the efficiency of optimization. As a result, the choice of modeling method should be very important [18]. The main categories of model include first-principle model, data-based (empirical) model and hybrid model. As an early preliminary study, the underlying mechanism of $\mathrm{CO}$ catalytic oxidation by $\mathrm{Au}-\mathrm{ZnO} / \mathrm{Al}_{2} \mathrm{O}_{3}$ is not fully clear. So first-principle model and hybrid model, which are based on mechanism, are not able to be used in this study. As its name suggests, data-based modeling is based simply on all available data. So the applications of data-based model are more widely, include all cases that have enough data.

The most important requirement for off-line data-based modeling method is the model accuracy and predictive reliability. For an uninvestigated process, implementation of traditional polynomial regression is inappropriate, since it performs poor for complex non-linear situation. In addition, polynomial regression has the over-fitting problem which needs to be addressed by using additional techniques (e.g. regularization, cross-validation). There are several approaches which can handle complex non-linear case, such as artificial neural network (ANN) [19], least-squares support vector machines (LS-SVMs) [20] and Gaussian process regression (GPR) [9]. They all attain wonderful model accuracy. However, GPR attains shorter computation time. As a result, GPR was adopted as the modeling method in this study and the details can be found in [5].

\subsection{Optimization}

In our former study [5], a rapid optimization method was implemented to handle the balance between predictive mean and variance. However, the maximization of expected improvement (EI) [21] approach is not suitable for this study, since the existing highest reactivity during DoE phase was already very high (99\%). Alternatively, a classic solution was adopted for this situation. With the current GPR model which developed with all 
available data, the data point $\mathbf{x}^{*}$ with highest predictive response $(\hat{y})$ was selected. After conducting experiment on $\mathbf{x}^{*}$ and obtain its real response $(y)$, the difference is compared as $\Delta y=|\hat{y}-y|$. This optimization procedure is an iterative one, that terminated when $\Delta y$ is small enough.

\subsection{Sensitivity Analysis}

For catalytic chemistry, as same important as response optimization, we also wondering how the factors and their interactions affect the performance of the process. A straight forward way is to test them with appropriate characterization techniques, like X-ray Diffraction (XRD) and transmission electron microscopy (TEM). However, for complex process which have more than three factors, the interactions are hardly to be evaluated purely based on characterization techniques [22]. Sensitivity analysis (SA), a class of statistical methods, is widely used for main effects and interactions analysis with limited data. By many works, their applications have been proved as stable and reliable [23-26]. In addition, the cost of funding and time by characterization techniques are much higher than those of using SA methods.

SA methods can be divided into two classes: local SA and global SA. Local SA depends on the derivatives of response against factors, on a fixed nominal point. For this reason, local SA methods cannot handle non-linear process [27]. By comparison, global SA considers the entire design space, giving a reliable picture of the process being investigated. Global SA approaches can be classified into screening methods, regression-based methods and variance-based methods [28]. Among them, variance-based methods are most widely used due to their reliable performance. In addition, the other two methods do not naturally provide sensitivity indices for high order interaction. Sobol' method was selected from the pool of variance-based methods, due to its superior performance and low computation cost [12]. Since SA indices cannot show whether the effect is positive or negative, a graphical SA method was applied to support. The details of Sobol' and the graphical SA methods can be checked in [22]. 


\section{Experimental}

\subsection{Catalyst Synthesis}

The chemicals involved in catalyst synthesis include $\mathrm{HAuCl}_{4} \cdot 3 \mathrm{H}_{2} \mathrm{O}(\geq 49.0 \% \mathrm{Au}$ basis, Sigma-Aldrich), $\mathrm{Zn}\left(\mathrm{NO}_{3}\right)_{2} \cdot 6 \mathrm{H}_{2} \mathrm{O}\left(98 \%\right.$, Sigma-Aldrich), $\mathrm{Al}\left[\mathrm{OCH}\left(\mathrm{CH}_{3}\right)_{2}\right]_{3}(\geq 98 \%$, Aldrich), and urea (98\%, Aldrich).

In this study, catalysts were prepared with an one-step method [5]. For every batch, powder of $\mathrm{Al}\left[\mathrm{OCH}\left(\mathrm{CH}_{3}\right)_{2}\right]_{3}$ was weighted and then added into a $50 \mathrm{~mL}$ round-bottomed flask, followed by adding $20 \mathrm{~mL}$ deionized (DI) water. After calculated amount of $\mathrm{HAuCl}_{4}$ and $\mathrm{Zn}\left(\mathrm{NO}_{3}\right)_{2}$ solutions were dropped in, and sufficient amount of urea were added. Subsequently, the mixture was kept at $90{ }^{\circ} \mathrm{C}$ for $6 \mathrm{~h}$, with magnetic stirred. Then the flask was cooled down to room temperature for overnight. The precipitate was washed with DI water for three times, and then kept in $100{ }^{\circ} \mathrm{C}$ oven for overnight. Before reaction, two-steps pretreatment were applied: (1) calcination with air for $4 \mathrm{~h}$, and $2{ }^{\circ} \mathrm{C} / \mathrm{min}$ heating rate; (2) reduction with hydrogen for $3 \mathrm{~h}$, and $5{ }^{\circ} \mathrm{C} / \mathrm{min}$ heating rate. For the two pretreatment steps, the heating temperatures were decided according to DoE and further optimization method.

\subsection{Reactivity Test}

The reactivities of catalysts were tested with a fixed-bed flow reactor, while the amount of catalyst varies by following DoE and optimization method. The gas flow rate of two reactants, $\mathrm{CO}$ and $\mathrm{O}_{2}$, was set as $2 \mathrm{~mL} / \mathrm{min}$ and $10 \mathrm{~mL} / \mathrm{min}$, respectively. The flow rate of $\mathrm{He}$, which acts as the carrier gas, was set to $50 \mathrm{~mL} / \mathrm{min}$. The product mixture was analyzed with an online GC $(6890 \mathrm{~N}$, Agilent Technologies), the equipped column was a $10 \mathrm{ft} \times 1 / 8$ in $\times 2.1 \mathrm{~mm}$ 100/120 Carbosieve SII column. The CO conversion was calculated with area of corresponding GC peaks.

\subsection{Characterization Techniques}

Powder X-ray diffraction (XRD) patterns were recorded on a Bruker D8 advance powder 
diffractometer at ambient conditions, using filtered $\mathrm{Cu}-\mathrm{K} \alpha$ radiation source $(\lambda=1.54056$ $\AA$ ) operated at $40 \mathrm{kV}$ and $40 \mathrm{~mA}$. Diffraction data were collected from 10 to $80^{\circ}(2 \theta)$ at a scanning speed of $2 \% \mathrm{~min}$. Prior to the test, samples were dried at $100{ }^{\circ} \mathrm{C}$ overnight.

Transmission electron microscopy (TEM) measurements were performed on JEOL JEM-1400 system operated at $100 \mathrm{kV}$. Before observation, the samples were suspended in ethanol and dispersed on a holey carbon-coated $\mathrm{Cu}$ grid.

\section{Results and discussion}

\subsection{Overall Optimization}

Considering the whole procedure of catalyst preparation, pretreatment and reaction, five factors were selected for optimization purpose: $x_{1}$ (weight loading of Au), $x_{2}$ (weight loading of $\mathrm{ZnO}), x_{3}$ (calcination temperature), $x_{4}\left(\mathrm{H}_{2}\right.$ reduction temperature), $x_{5}$ (catalyst weight). The ranges of these factors were set as $1-3 \%, 1-5 \%, 200-500{ }^{\circ} \mathrm{C}, 100-300$ ${ }^{\circ} \mathrm{C}$ and 20-100 mg, respectively [5]. According to former experience, 16 experiments were designed following HSS method and listed in Table 1. In this work, the resultant CO reactivity $y(\%)$ is the average of conversions under $30,50,70$ and $90{ }^{\circ} \mathrm{C}$. The detailed conversions of each catalyst are shown in Figure 1.

Table 1. Designed experiments and resultant CO reactivity $y(\%)$ : the 1st DoE.

\begin{tabular}{ccccccc}
\hline Run No. & $x_{1}$ & $x_{2}$ & $x_{3}$ & $x_{4}$ & $x_{5}$ & $y(\%)$ \\
\hline 1 & 2.88 & 3 & 400 & 260 & 89 & 82.75 \\
2 & 2.75 & 4 & 300 & 220 & 77 & 98.75 \\
3 & 2.63 & 2 & 467 & 180 & 66 & 98.75 \\
4 & 2.5 & 4.5 & 367 & 140 & 54 & 95.5 \\
5 & 2.38 & 2.5 & 267 & 292 & 43 & 74.25
\end{tabular}




\begin{tabular}{ccccccc}
6 & 2.25 & 3.5 & 433 & 252 & 31 & 54 \\
7 & 2.13 & 1.5 & 333 & 212 & 98 & 73 \\
8 & 2 & 4.75 & 233 & 172 & 87 & 79.5 \\
9 & 1.88 & 2.75 & 489 & 132 & 76 & 85.5 \\
10 & 1.75 & 3.75 & 389 & 284 & 64 & 70.25 \\
11 & 1.63 & 1.75 & 289 & 244 & 53 & 64 \\
12 & 1.5 & 4.25 & 456 & 204 & 41 & 35.25 \\
13 & 1.38 & 2.25 & 356 & 164 & 30 & 35 \\
14 & 1.25 & 3.25 & 256 & 124 & 97 & 96.75 \\
15 & 1.13 & 1.25 & 422 & 276 & 85 & 64.5 \\
16 & 1 & 4.88 & 322 & 236 & 74 & 29.25 \\
\hline
\end{tabular}
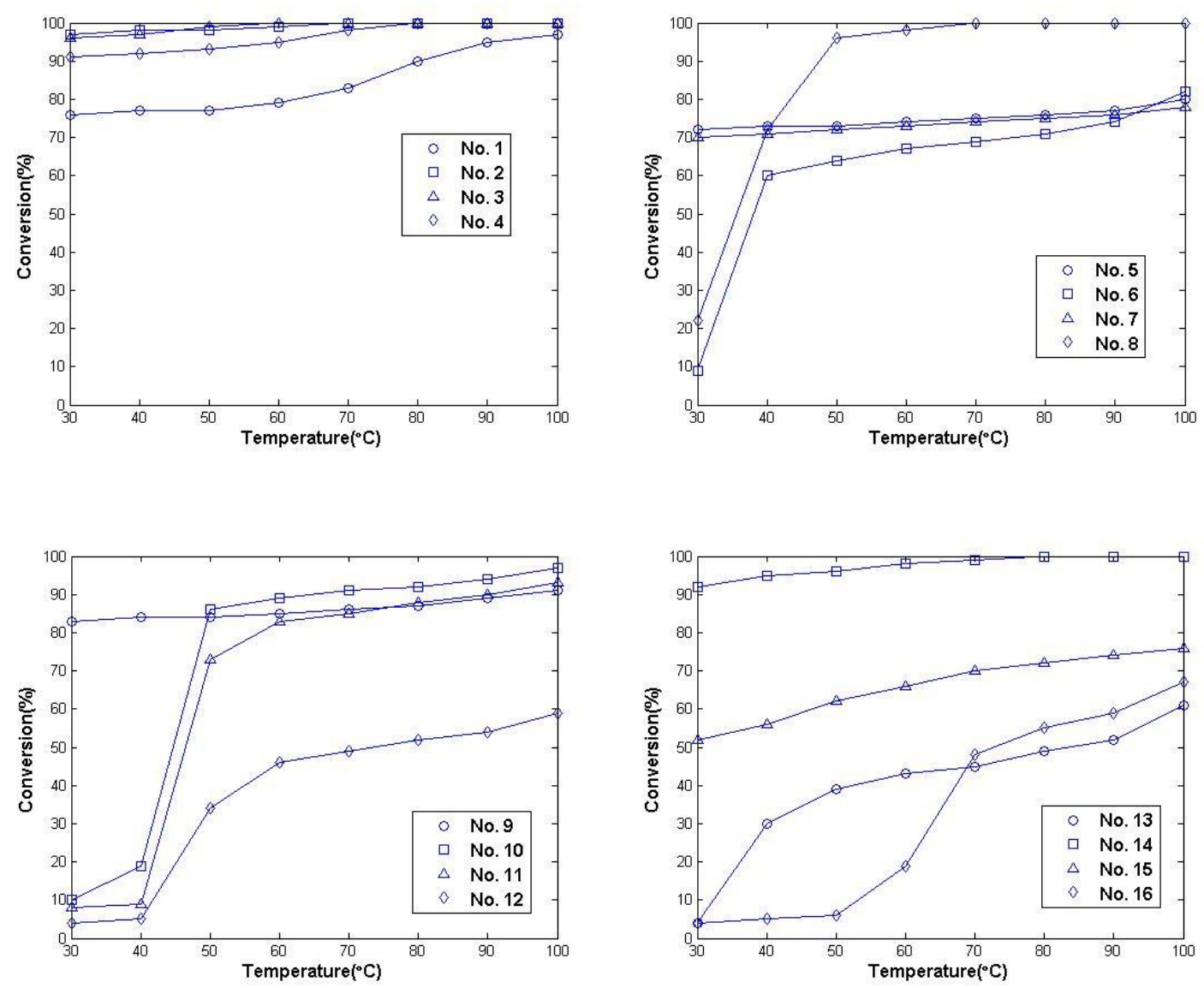
Figure 1. Corresponding conversion of catalysts in 1 st DoE.

In Table 1, there was already some catalysts shown extremely high reactivity $(98.75 \%$ for No. 2 and 3). For this reason, further optimizing for reactivity solely did not has significant meaning, since No.2 or 3 can satisfy the requirement if customer only needs high performance catalyst. So in this study, we alternatively tried to decrease the loading of $\mathrm{Au}$ while keeping high reactivity. For this reason, the new range for $x_{1}$ was set to $1-2 \%$, for lower $\mathrm{Au}$ loading. The ranges of $x_{2}-x_{5}$ were set as $3-5 \%, 200-300{ }^{\circ} \mathrm{C}, 100-200{ }^{\circ} \mathrm{C}$ and 50-100 mg, respectively, to keep high reactivity. Considering the region with high reactivity (higher than 90\%) do not have enough data points (four only), the 2nd DoE step was added to help explore the high-reactivity region, with eight points designed by HSS. The according data are shown in Table 2 with corresponding reactivities, and the detailed conversions of each catalyst are shown in Figure 2.

Table 2. Designed experiments and resultant CO reactivity $y \quad(\%)$ : the 2 nd DoE.

\begin{tabular}{ccccccc}
\hline Run No. & $x_{1}$ & $x_{2}$ & $x_{3}$ & $x_{4}$ & $x_{5}$ & $y(\%)$ \\
\hline 17 & 1.88 & 4 & 267 & 180 & 93 & 99 \\
18 & 1.75 & 4.5 & 233 & 160 & 86 & 99.25 \\
19 & 1.63 & 3.5 & 289 & 140 & 79 & 98 \\
20 & 1.5 & 4.75 & 256 & 120 & 71 & 77.25 \\
21 & 1.38 & 3.75 & 222 & 196 & 64 & 91 \\
22 & 1.25 & 4.25 & 278 & 176 & 57 & 80.5 \\
23 & 1.13 & 3.25 & 244 & 156 & 99 & 99 \\
24 & 1 & 4.88 & 211 & 136 & 92 & 94.75 \\
\hline
\end{tabular}



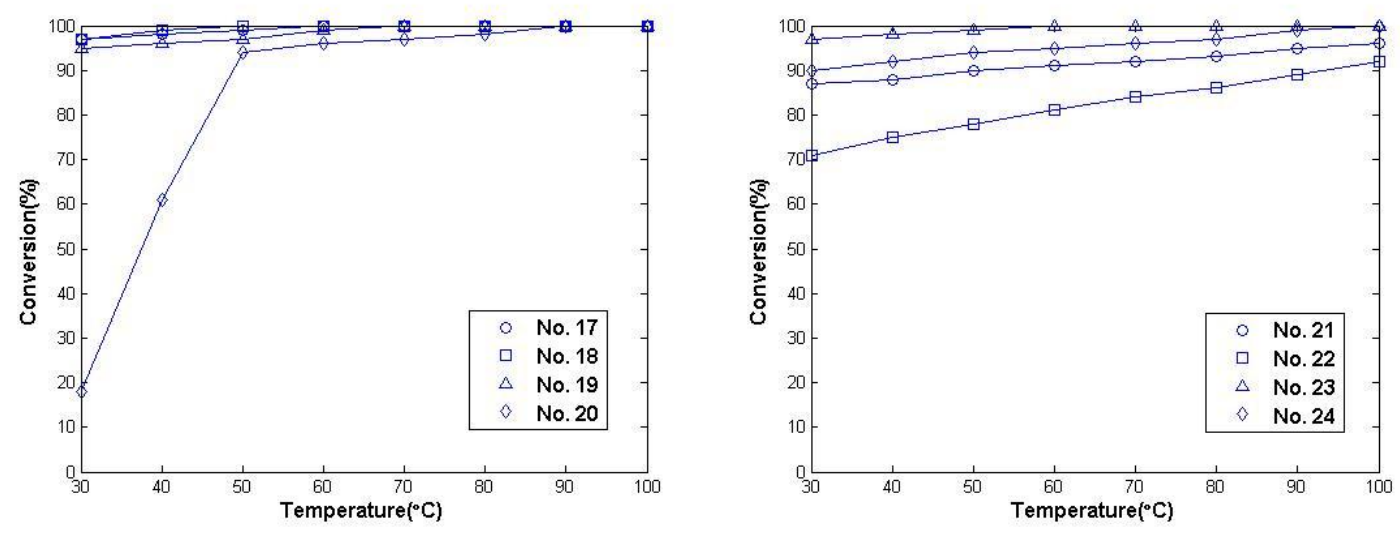

Figure 2. Corresponding conversion of catalysts in 2nd DoE.

After obtaining these eight points, the study entered the phase of iterative optimization. Since low Au loading also can achieve extremely high reactivity (No. 23), the loading of Au was fixed to $1 \%$ during optimization. With the GPR model developed from all data points, the point with highest predictive mean was demonstrated as $100 \mathrm{mg}$ of $1.0 \% \mathrm{Au}-4.1 \% \mathrm{ZnO} / \mathrm{Al}_{2} \mathrm{O}_{3}$ catalyst with $220{ }^{\circ} \mathrm{C}$ calcination and $100{ }^{\circ} \mathrm{C}$ reduction (No.25). Its reactivity was predicted as $99.87 \%$ and confirmed to be $100 \%$. Since the variance was very small, further test can be ignored. As this catalyst gave full conversion from $30{ }^{\circ} \mathrm{C}$ to $100{ }^{\circ} \mathrm{C}$, the figure of details is not given.

\subsection{Sensitivity Analysis}

A GPR model was developed based on the entire 25 experiments, with four pre-study data. The results of these four pre-study experiments are listed in Table 3 and Figure 3. This model was used to elucidate the impact of all five factors on catalyst performance: $x_{1}$ (weight loading of $\mathrm{Au}$ ), $x_{2}$ (weight loading of $\mathrm{ZnO}$ ), $x_{3}$ (calcination temperature), $x_{4}$ $\left(\mathrm{H}_{2}\right.$ reduction temperature), $x_{5}$ (catalyst weight).

Table 3. Designed experiments and resultant CO reactivity $y(\%)$ : Pre-study.

$\begin{array}{lllllll} & & x_{2} & x_{3} & x_{5} & y(\%)\end{array}$




\begin{tabular}{ccccccc}
\hline Pre-01 & 1 & 3 & 275 & 150 & 50 & 74.75 \\
Pre-02 & 1 & 3 & 250 & 175 & 50 & 77 \\
Pre-03 & 1 & 3 & 225 & 125 & 50 & 82.5 \\
Pre-04 & 1 & 3 & 200 & 188 & 50 & 73.5 \\
\hline
\end{tabular}

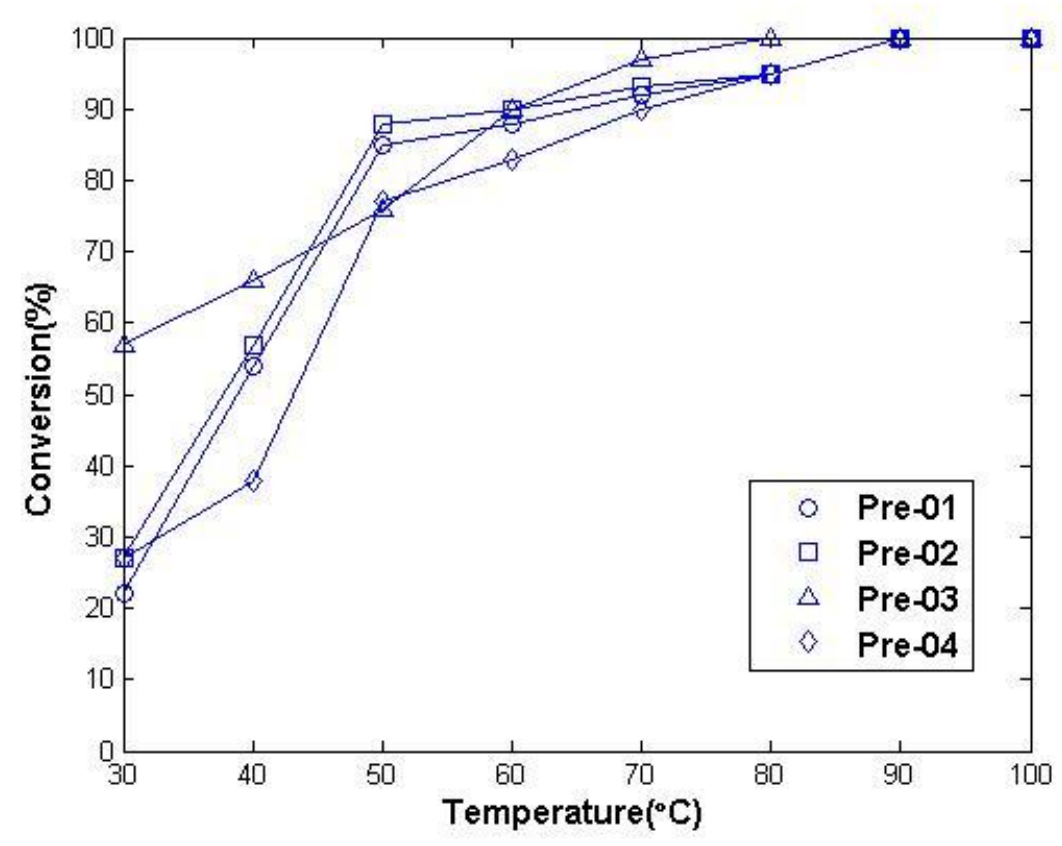

Figure 3. Corresponding conversion of catalysts in pre-study.

It was found that 10,000 Monte Carlo samples were sufficient for accurate approximation of the integrals while being computationally manageable [29]. The main effects due to the five factors are shown in Table 4 (both $S_{i}$ and $S_{T_{i}}$ ). SIs quantifies the magnitude of the factors' influence, but not the trend. A graphical illustration of the main effects versus various factors, as shown in Figure 4, is a useful complement to the SIs. Note the factors are scaled to lie in the range $[0,1]$ in Figure 4.

Table 4. SIs of the main effects.

\begin{tabular}{llllll}
\hline Factor & $x_{1}$ & $x_{2}$ & $x_{3}$ & $x_{4}$ & $x_{5}$ \\
\hline
\end{tabular}




\begin{tabular}{cccccc}
\hline$S_{i}$ & 0.334 & 0.037 & 0.156 & 0.117 & 0.232 \\
$S_{T_{i}}$ & 0.416 & 0.206 & 0.284 & 0.153 & 0.343 \\
\hline
\end{tabular}

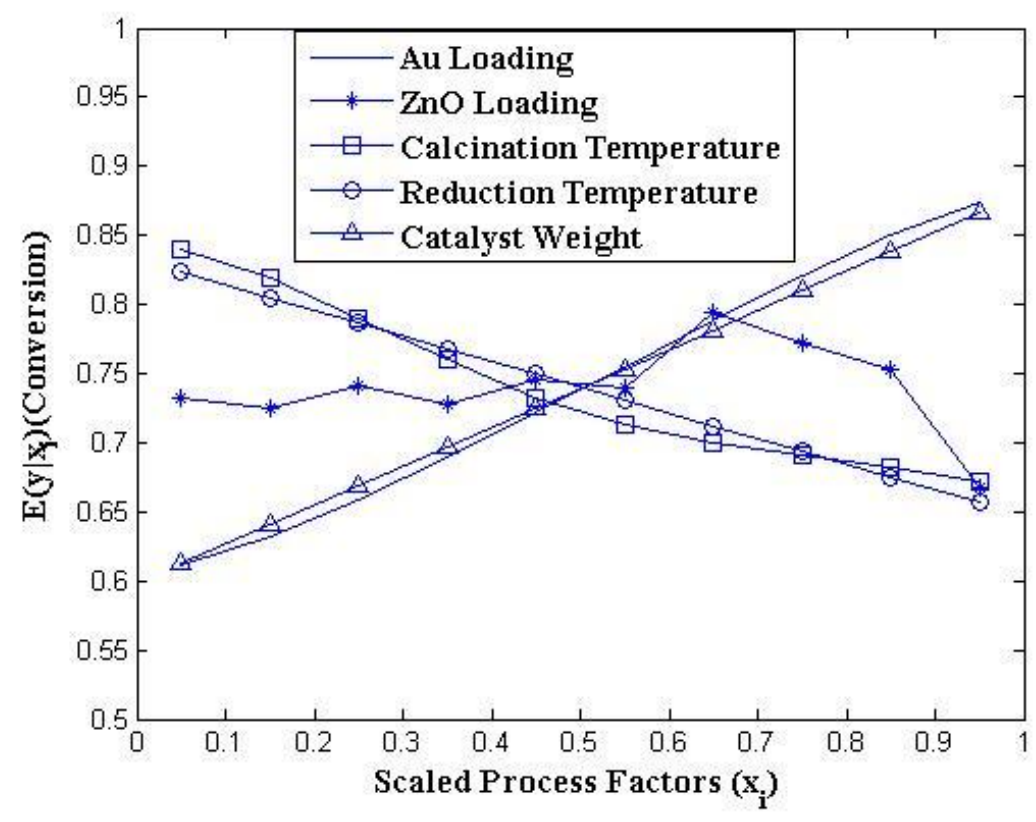

Figure 4. The main effect $E\left(y \mid x_{i}\right)$ against $x_{i}$ for each process factor.

With Table 4 and Figure 4, the main effect for each factor can receive a clear demonstration. Au loading $\left(x_{1}\right)$ attains highest impact $\left(S_{1}=0.334\right)$, and the graph of $E\left(y \mid x_{1}\right)$ favors a higher Au loading. It is obviously since Au is the active site of catalyst, which was confirmed from our former work. Catalyst Weight $\left(x_{5}\right)$ has the second highest impact, and the graph of $E\left(y \mid x_{5}\right)$ favors a higher catalyst weight. It can be demonstrated as higher catalyst weight leads to longer contact time, further leads to better performance. Calcination temperature $\left(x_{3}\right)$ and reduction temperature $\left(x_{4}\right)$ also shown significant main effect, however, the graphs of $E\left(y \mid x_{3}\right)$ and $E\left(y \mid x_{4}\right)$ shown that higher temperature leads to lower reactivity. The preferences of $E\left(y \mid x_{3}\right)$ and $E\left(y \mid x_{4}\right)$ can be confirmed by the 
results of No. 25, and would be demonstrated at the end of this section. The main effect of $\mathrm{ZnO}$ loading $\left(x_{2}\right)$ is weak and its trend is complicated. The quick drop of $E\left(y \mid x_{2}\right)$ of [0.7, 1] may be due to the overdose of $\mathrm{ZnO}$, which may cover the surface of Au particle and then decrease the contact of Au particle and reactants.

Different from $S_{i}, S_{T_{i}}$ includes not only the main effect but also the effects from related interactions. From the differences between $S_{i}$ and $S_{T_{i}}$ shown in Table 4, we can conclude that there are important effects of $x_{2}$-related interactions and $x_{3}$-related interactions. In addition, the sum of five main indices is 0.876 , suggesting that the interactions would account for $1--0.876=12.4 \%$ of the total variation. As a result, further analysis should be conducted to assess the interaction terms.

Firstly the second order interactions were calculated and listed in Table 5. Then we found that the sum of main effects and second order interactions is 0.994 , which implies that investigation on higher-order interactions was not necessary. From Table 5, the conclusion can be obtained that $\mathrm{Au}-\mathrm{ZnO}$ interaction $\left(S_{1,2}=0.054\right)$ and $\mathrm{ZnO}$-Calcination interaction $\left(S_{2,3}=0.053\right.$ ) have significant effluence on reaction performance. From Figure 5 and 6 , the trend for these two interactions are strong and complicated, not simply positive or negative. The strong $\mathrm{Au}-\mathrm{ZnO}$ interaction confirmed the conclusion from our former work [5], and would be demonstrated in mechanism later.

Table 5. SIs of the second order interactions.

\begin{tabular}{ccccc}
\hline$S_{i, j}$ & $x_{1}$ & $x_{2}$ & $x_{3}$ & $x_{4}$ \\
\hline$x_{2}$ & 0.054 & & & \\
$x_{3}$ & 0.000 & 0.053 & & \\
$x_{4}$ & 0.011 & 0.000 & 0.000 & \\
$x_{5}$ & 0.000 & 0.000 & 0.000 & 0.000 \\
\hline
\end{tabular}




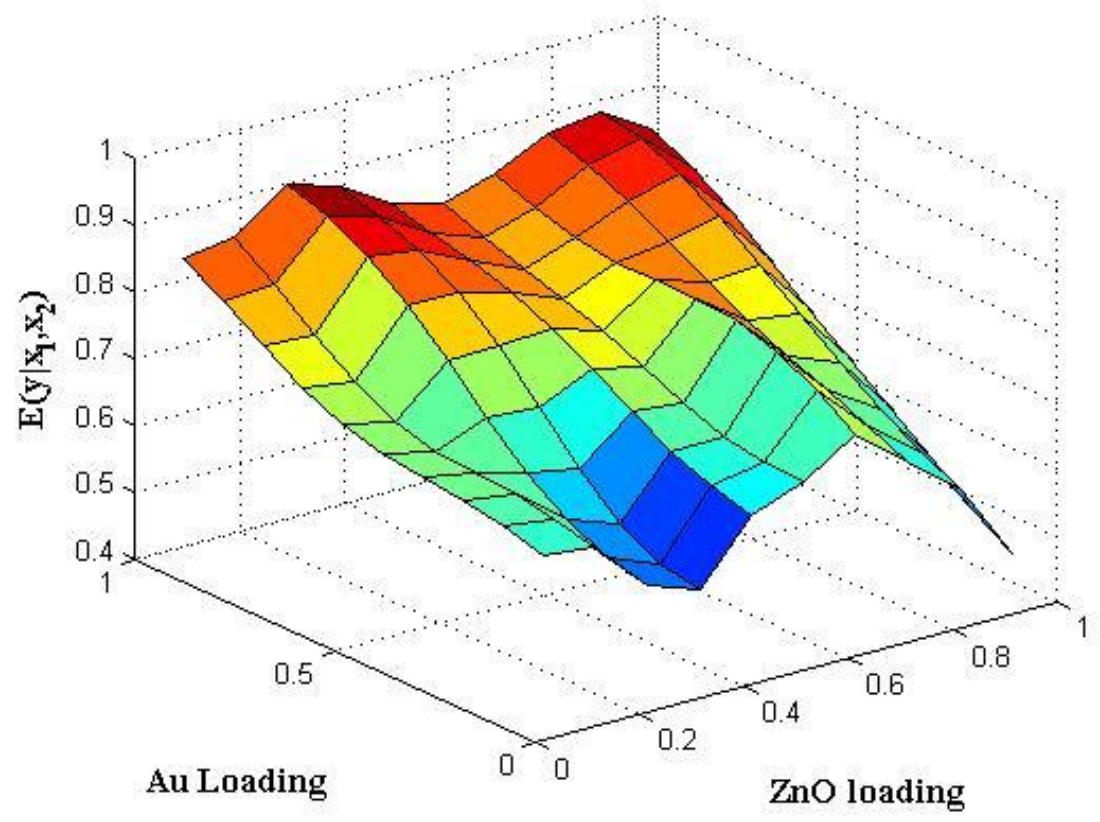

Figure 5. The expectation, $E\left(y \mid x_{i}, x_{j}\right)$, against Au loading $\left(x_{1}\right)$ and $\mathrm{ZnO}$ loading $\left(x_{2}\right)$. Process factors are scaled.

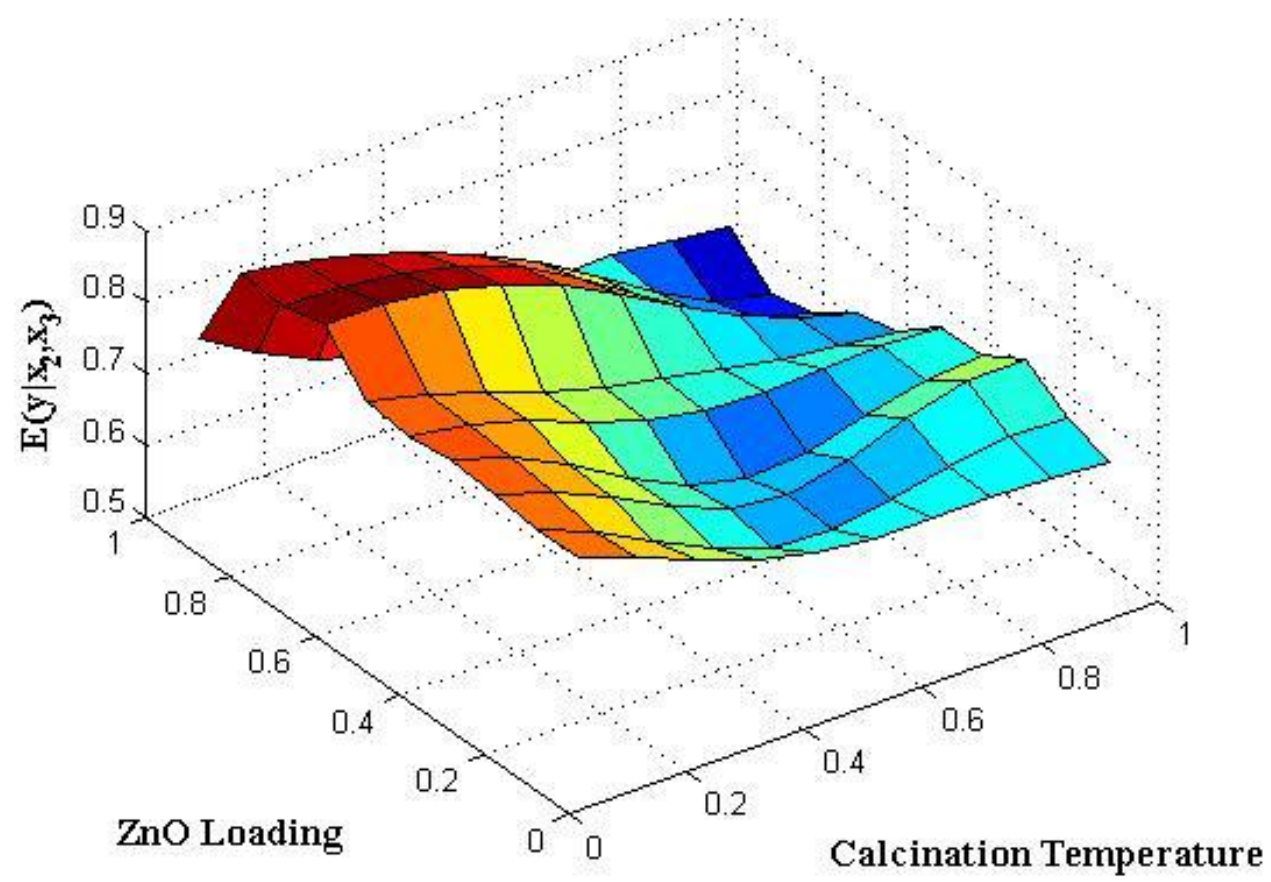

Figure 6. The expectation, $E\left(y \mid x_{i}, x_{j}\right)$, against $\mathrm{ZnO}$ loading $\left(x_{2}\right)$ and calcination temperature $\left(x_{3}\right)$. Process factors are scaled. 
For the reaction mechanism of catalytic CO oxidation, a widely accepted theory is LH mechanism on $\mathrm{Au}$ surface [30]. As Qian demonstrated, oxygen adatoms were generated from direct oxygen dissociation which adsorbed $\mathrm{CO}$ to form $\mathrm{CO}_{2}$. In this mechanism, oxygen dissociation step is the rate-limiter and the Au particle size has a significant influence on it. There are also some articles that support this influence with experimental results $[31,32]$. According to this mechanism, some aforementioned conclusions from sensitivity analysis can be demonstrated.

For $\mathrm{Au}-\mathrm{ZnO}$ interaction, presence of $\mathrm{ZnO}$ can deduce the size of $\mathrm{Au}$ particles [33], further improves catalysis reactivity. In addition, presence of $\mathrm{Au}$ grows the oxygen vacancies on $\mathrm{ZnO}$ [34], also improves the catalysis reactivity. For calcination temperature, lower temperature can lead to smaller Au crystallites, which further lead to higher catalysis reactivity. For reduction temperature, high temperature may lead to particle aggregation, which deduces catalysis reactivity [35].

\subsection{Characterization}

As mentioned in Section 4.2, the effects of calcination and $\mathrm{H}_{2}$ reduction temperature are important for the catalytic $\mathrm{CO}$ oxidation process with $\mathrm{Au}-\mathrm{ZnO} / \mathrm{Al}_{2} \mathrm{O}_{3}$. For further study, four catalysts were designed as Table 6 to demonstrate these two temperature effects. In this table, the corresponding reactivity of these catalysts is also listed. These catalysts were labeled as $\mathrm{CR}$ catalysts, that $\mathrm{CR}$ stand for $\mathrm{C}$ (alcination) and $\mathrm{R}$ (eduction). From the differences of reactivity, the effects of calcination and reduction temperature can be initially justified.

Table 6. SIs of the second order interactions.

\begin{tabular}{ccccccc}
\hline Run No. & $x_{1}$ & $x_{2}$ & $x_{3}$ & $x_{4}$ & $x_{5}$ & $y(\%)$ \\
\hline $\mathrm{C}(-) \mathrm{R}(-)$ & 1 & 3 & 200 & 100 & 50 & 82.34 \\
$\mathrm{C}(-) \mathrm{R}(+)$ & 1 & 3 & 200 & 200 & 50 & 72.48 \\
$\mathrm{C}(+) \mathrm{R}(-)$ & 1 & 3 & 300 & 100 & 50 & 73.77 \\
$\mathrm{C}(+) \mathrm{R}(+)$ & 1 & 3 & 300 & 200 & 50 & 63.49 \\
\hline
\end{tabular}


To further validate these effects and investigate the properties simultaneously, two characterization techniques were applied. The patterns of XRD results are shown in Fig. 7, where the peaks at $2 \theta=38^{\circ}$ and $2 \theta=64^{\circ}$ correspond to the (111) and (220) polymorph of $\mathrm{Au}$, respectively $[32,36]$. Due to the low Au loading, the strength of signal was too weak to calculate particle size. The peaks of $\mathrm{ZnO}$ species do not presented in the patterns, suggests that they were dispersed within tiny size that XRD cannot detect.

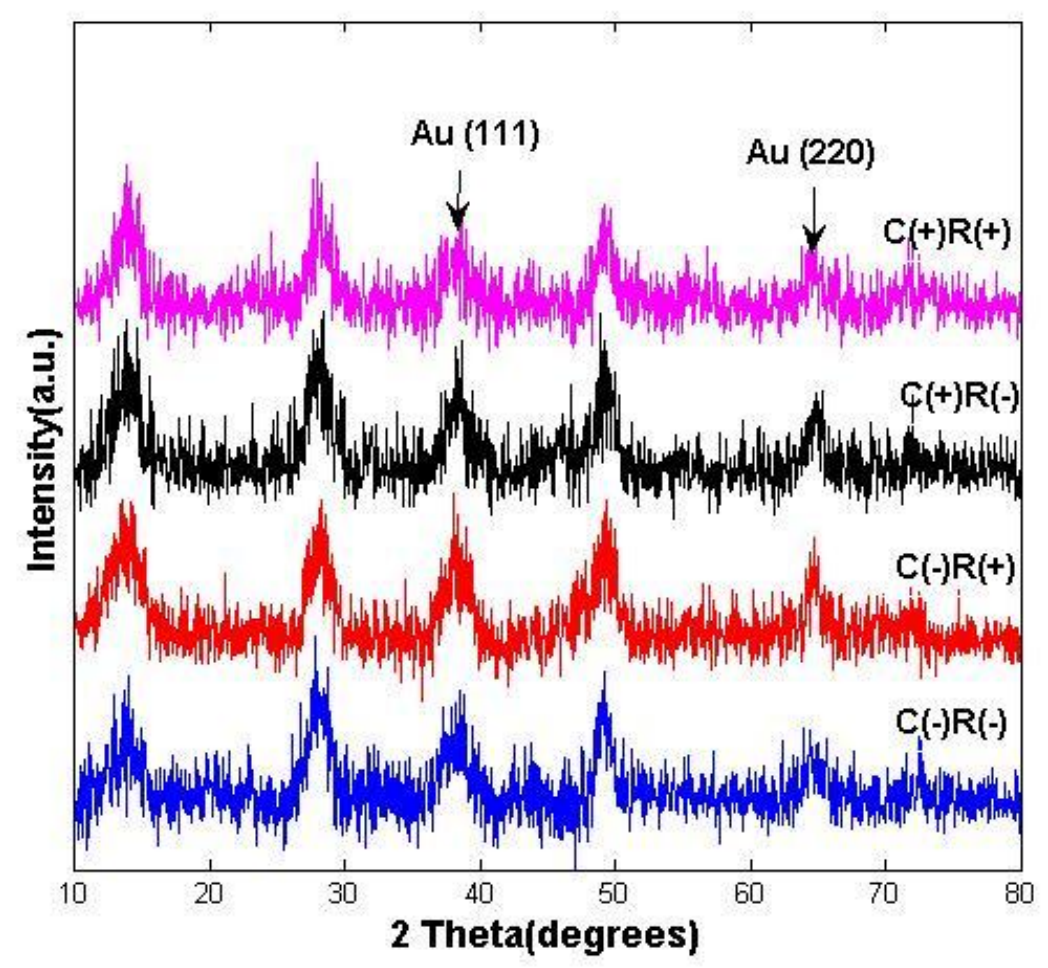

Figure 7. XRD patterns of CR catalysts.

TEM images of these four catalysts were also collected and compared. Unlike the TEM images in our former study [5], particles on CR catalysts are too small to calculate particle size. Although particles are concentrated in some area, they can be demonstrated to be uniformly scattered on support. 

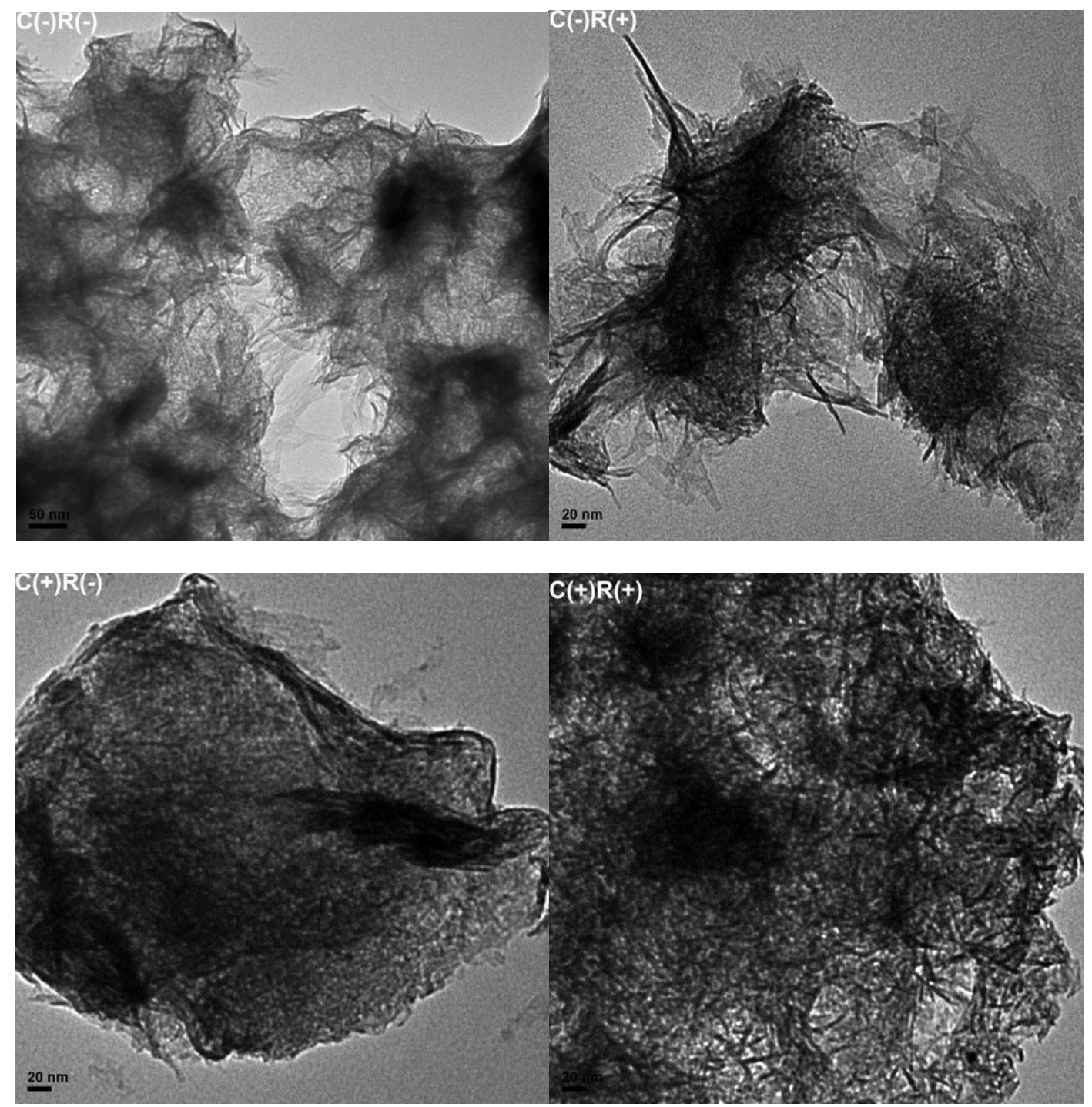

Figure 8. TEM images of CR catalysts.

\section{Conclusions}

In this study, our model-aided catalyst design and optimization approach was applied to $\mathrm{Au}-\mathrm{ZnO} / \mathrm{Al}_{2} \mathrm{O}_{3}$ catalyzed $\mathrm{CO}$ oxidation. Not only the loading of $\mathrm{Au}$ and $\mathrm{ZnO}$, but also three process parameters (calcination temperature, $\mathrm{H}_{2}$ reduction temperature and catalyst weight) were considered together. In addition, the cost of catalyst itself was controlled at a low level. With totally 25 experiments, the system was optimized to $100 \mathrm{mg} 1.0 \% \mathrm{Au}-4.1 \% \mathrm{ZnO} / \mathrm{Al}_{2} \mathrm{O}_{3}$ catalyst with $220{ }^{\circ} \mathrm{C}$ calcination and $100{ }^{\circ} \mathrm{C}$ reduction. It can be demonstrated that the optimization of process parameters simultaneously improve catalyst composition. 
For analysis of the main effects and interactions in this process, a global sensitivity analysis approach was implemented. For main effects, all factors exclude $\mathrm{ZnO}$ loading play significant role, and range as Au loading > catalyst weight $>$ calcination temperature $>\mathrm{H}_{2}$ reduction temperature, by importance. For interactions, there are two important interactions which affect the response: (1) $\mathrm{Au}-\mathrm{ZnO}$ interaction; and (2) $\mathrm{ZnO}$-Calcination interaction. Some of the significant effects were confirmed from thermodynamic mechanism, experimental results or characterization techniques, while others need further investigation.

\section{References}

1. Kim H Y, Han S S, Ryu J H, and Lee H M. J. Phys. Chem. C, 2010. 114: 3156.

2. Kim Y H, Park E D, Lee H C, Lee D, and Lee K H. Catal. Today, 2009. 146: 253.

3. Somodi F, Borbath I, Hegedus M, Lazar K, Sajo I E, Geszti O, Rojas S, Fierro J L G, and Margitfalvi J L. Appl. Surf. Sci., 2009. 256: 726.

4. $\quad$ Bion N, Epron F, Moreno M, Marino F, and Duprez D. Top. Catal., 2008. 51: 76.

5. Yan W, Chen Y, Yang Y, and Chen T. Catal. Today, 2011. 174: 127.

6. Myers R H and Montgomery D C, Response Surface Methodology: Process and Product in Optimization Using Designed Experiments: John Wiley \& Sons, Inc. 1995. 728.

7. Kalagnanam J R and Diwekar U M. Technometrics, 1997. 39: 308.

8. Chen V C P, Tsui K L, Barton R R, and Meckesheimer M. Iie Transactions, 2006. 38: 273.

9. Williams C K I and Rasmussen C E, Gaussian processes for machine learning: MIT Press. 2006.

10. Yuan J, Wang K S, Yu T, and Fang M L. International Journal of Machine Tools \& Manufacture, 2008. 48: 47.

11. Sobol I M. Mathematical Modeling and Computational Experiment, 1993. 14: 407.

12. Saltelli A and Sobol I M. Reliability Engineering \& System Safety, 1995. 50: 225.

13. Lopez I, Valdes-Solis T, and Marban G. Int. J. Hydrogen Energy, 2008. 33: 197.

14. Mandenius C F and Brundin A. Biotechnol. Prog., 2008. 24: 1191.

15. Mohan S V, Raghavulu S V, Mohanakrishna G, Srikanth S, and Sarma P N. Int. J. Hydrogen Energy, 2009. 34: 216.

16. Fang K T, Lin D K J, Winker P, and Zhang Y. Technometrics, 2000. 42: 237.

17. McKay M D, Beckman R J, and Conover W J. Technometrics, 2000. 42: 55.

18. Omata K, Kobayashi Y, and Yamada M. Energy Fuels, 2009. 23: 1931.

19. Cheng B and Titterington D M. Statistical Science, 1994. 9: 2.

20. Thissen U, Ustun B, Melssen W J, and Buydens L M C. Anal. Chem., 2004. 76: 3099.

21. Jones D R. Journal of Global Optimization, 2001. 21: 345.

22. Yan W, Guo Z, Jia X, Kariwala V, Chen T, and Yang Y. Chem. Eng. Sci., 2012. 76: 26.

23. Scott V, Kettle H, and Merchant C J. Ocean Science, 2011. 7: 405.

24. Haaker M P R and Verheijen P J T. Chem. Eng. Res. Des., 2004. 82: 591.

25. Tchuenche J M, Khamis S A, Agusto F B, and Mpeshe S C. Acta Biotheoretica, 2011. 59: 1. 
26. Degerman M, Westerberg K, and Nilsson B. Chem. Eng. Technol., 2009. 32: 1195.

27. Saltelli A, Ratto M, Andres T, Campolongo F, Cariboni J, Gatelli D, Saisana M, and Tarantola S, Global Sensitivity Analysis. The Primer: John Wiley \& Sons, Ltd. 2008. i.

28. Confalonieri R, Bellocchi G, Bregaglio S, Donatelli M, and Acutis M. Ecological Modelling, 2010. 221: 1897.

29. Chen T and Yang Y H. Chemom. Intell. Lab. Syst., 2011. 107: 116.

30. Qian K, Huang W X, Fang J, Lv S S, He B, Jiang Z Q, and Wei S Q. J. Catal., 2008. 255 : 269.

31. Naknam P, Luengnaruemitchai A, and Wongkasemjit S. Energy Fuels, 2009. 23: 5084.

32. Chang L H, Chen Y W, and Sasirekha N. Ind. Eng. Chem. Res., 2008. 47: 4098.

33. Chang F W, Lai S C, and Roselin L S. Journal of Molecular Catalysis a-Chemical, 2008. 282 : 129.

34. Laguna O H, Centeno M A, Romero-Sarria F, and Odriozola J A. Catal. Today, 2011. 172: 118.

35. Chen X R, Zou H B, Chen S Z, Dong X F, and Lin W M. J. Nat. Gas Chem., 2007. 16: 409.

36. Khanderi J, Hoffmann R C, Engstler J, Schneider J J, Arras J, Claus P, and Cherkashinin G. Chemistry-a European Journal, 2010. 16: 2300. 